\title{
Estimation of the Heritability of Latent Variables Which Are Included in a Structural Model for Metabolic Syndrome
}

\author{
Rainer Koch ${ }^{a}$ Ulrich Julius ${ }^{b}$ Werner Jaross ${ }^{c}$ Hans-Egbert Schröder ${ }^{d}$ \\ a Institute for Medical Informatics and Biometrics, b Institute and Policlinic for Clinical Metabolic Research, \\ cInstitute for Clinical Chemistry and Laboratory Medicine, and dMedical Clinic and Policlinic III, \\ Medical Faculty Carl Gustav Carus, University, of Technology, Dresden, Germany
}

\section{Key Words}

Metabolic syndrome - Multivariate phenotypic trait ·

Structural equation modeling $\cdot$ Heritability

\begin{abstract}
In a study looking for risk factors of atherosclerosis in families with combined hyperlipidemia and hypertension, clinical and biochemical data of 1,149 persons were analyzed to develop two hypothetical multivariate scores concerning the degree to which a patient is affected by the metabolic syndrome. The scores are based on a structural model for low-density cholesterol (LDL) and high-density cholesterol (HDL), triglycerides, uric acid, creatinine, glucose, insulin, systolic blood pressure and waist-to-hip ratio. Age, gender and body mass index were used for adjusting all variables. In segregation analyses of 42 pedigrees without using genotype information, estimations of the heritabilities and environmentally caused variance and covariance components were computed for the individual score values of the two latent factors. The first score shows a heritability of $42 \%$; the environment component disappeared. The score mainly reflects the HDL, LDL and triglyceride levels. The second score shows a heritability of $16 \%$ with an environment component of $7 \%$. It includes mainly insulin,
\end{abstract}

\section{KARGER}

Fax + 41613061234

E-Mail karger@karger.ch

www. karger.com

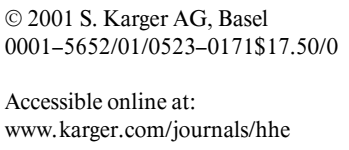

uric acid and creatinine. In the search for genetic causes, both scores could be a basis for further phenotypic classification of the metabolic syndrome.

Copyright @ 2001 S. Karger AG, Basel

\section{Introduction}

The results of previous search for genetic causes of the metabolic syndrome suggest polygenetic events. A convincing method to discriminate phenotypes for familial combined hyperlipidemia or more generally for the metabolic syndrome, which could qualify for systematic linkage analyses with some candidate genes or marker loci, has not been discovered yet. Existing classifications of lipid disorders [1] are predominantly based on lipid and apolipoprotein concentrations and partially include clinical findings. On linkage analysis, no single ultracentrifugation variable for the diagnosis of familial combined hyperlipidemia could reliably discriminate affected family members from nonaffected members [2]. There are various strategies to include several of such quantitative variables into the definition of phenotypes or into linkage analyses with the aim to increase the chances of finding linkages with genetic markers. Examples include cluster analyses of diagnostic parameters [3], explorative factor 
Table 1. Parameters used in the analyses, means and standard deviations (SD) in the sample

\begin{tabular}{|c|c|c|c|}
\hline Variables & & Mean & SD \\
\hline \multicolumn{2}{|l|}{ Triglycerides, mmol/l } & 1.72 & 1.49 \\
\hline $\log$ triglycerides & 1 & 0.335 & 0.609 \\
\hline HDL cholesterol, mmol/1 & 2 & 1.35 & 0.39 \\
\hline LDL cholesterol, mmol/1 & 3 & 3.41 & 1.30 \\
\hline Uric acid, $\mu \mathrm{mol} / 1$ & 4 & 318 & 87 \\
\hline Creatinine, $\mu \mathrm{mol} / 1$ & 5 & 88 & 18 \\
\hline \multicolumn{2}{|l|}{ Glucose, $\mathrm{mmol} / \mathrm{l}$} & 4.33 & 1.73 \\
\hline log glucose & 6 & 1.38 & 0.45 \\
\hline Insulin, nmol/1 & & 0.080 & 0.068 \\
\hline $\log$ insulin & 7 & -2.73 & 0.60 \\
\hline Systolic blood pressure & 8 & 132.2 & 19.8 \\
\hline Waist-to-hip ratio & 9 & 0.867 & 0.104 \\
\hline Age & & 41.5 & 17.0 \\
\hline \multicolumn{4}{|l|}{ Sex 601 males } \\
\hline Body mass index, $\mathrm{kg} / \mathrm{m}^{2}$ & 10 & 25.2 & 4.7 \\
\hline
\end{tabular}

analyses $[4,5]$, the development of path models for hypothetical relationships between the variables [6,7], and the estimation of genetic and environmental variance components [8-10]. Our approach combines methods of structural analysis with the estimation of genetic variance components. In a first step, the observed correlation matrix of the variables in the sample is reproduced by a linear structural model with two latent variables. In a second step, one of these latent variables is identified as a hypothetical genetic component by bivariate estimation of genetic and environmental variance components. The factor values of these latent variables can also be individually estimated for further persons and they indicate to which degree a person is affected with various lipid metabolism disorders. Thus, our approach describes a reduction in the dimension of the diagnostic variables to two latent variables and a subsequent bivariate estimation of the variance and covariance components of these latent variables. Alternatively to our approach, a continuous multivariate strategy could use the first principal component of the genetic covariance matrix of all original diagnostic variables to define the phenotype. However, this principal component could only be interpreted medically with difficulty and it would be less likely to be accepted as a phenotypic trait. In comparison, our approach has the advantage of the plastic model of relationships between the original variables and the ability of interpreting the latent variables as clinical score for the affect referring to the metabolic syndrome. Besides, considerable less parameters of the model have to be estimated in comparison to the continuous multivariate approach. In addition, the request for the first principal component to deliver maximal variance reproduction with the aim to make it unique, appears artificial in a physiological context.

A further advantage of the structural model is the integration of reliable knowledge about causal associations between biochemical parameters and further clinical and paraclinical parameters. As an example, the work of Chan et al. [7] can be given. In a causal path model, associations are described between glucose, triglycerides, insulin, urinary albumin, blood pressure, age, body mass index, waist-to-hip ratio and family history for patients with diabetes mellitus.

\section{Methods}

Step One: Structural Model for the Correlations and Estimation of the Scores

Data Collection. The sample for the development of the structural model was taken from the data bank of our atherosclerosis study group [11, 12]. Patients who have been cared for on an outpatient basis at our Lipid Clinic and at the Department of Internal Medicine have been included in the study on a voluntary basis (written consent). A known diabetes mellitus, a malignant or any other life-threatening disease were exclusion criteria. A systematic history was taken and blood sampling was only done in periods without relevant acute diseases or without severe illnesses or operations in the last months. Family members of these index patients have been invited to take part in the same program. The main target of the study was to identify families with hypertriglyceridemia or combined hyperlipidemia, and thus no medication has been discontinued. Blood samples were taken after an overnight fast. All 1,149 persons with a complete set of biochemical parameters were selected from the data bank. The sample consisted of 42 families with 308 members and 841 single persons: 601 males aged $42.4 \pm 16.9$ (range 5-86) years and 548 females aged $40.9 \pm 17.1$ (range 4-86) years. In an epidemiological perspective, the analysis is based on a cross-sectional survey of a mixed population consisting of affected index persons and optionally of their relatives and partners. We assume the same correlation structure between the variables for all members of this mixed population. This implies that the hypothetical genetic influences on a single individual only change the expected values of variables but not the association structures between variables. This assumption is possibly too restrictive in a medical sense, but it is unavoidable even in the continuous approach described above.

Laboratory Assays. Table 1 summarizes the variables used in the analysis. For all laboratory parameters, standard biochemical methods were used as published before [13], total serum cholesterol: CHOD-PAP (Boehringer Mannheim); serum triglycerides: GPOPAP (Boehringer Mannheim); high-density lipoprotein cholesterol (HDCL-C): precipitation of apolipoprotein-B-containing lipoproteins with polyphosphotungstate and magnesium ions; and cholester- 
ol determination (Boehringer Mannheim), low-density lipoprotein cholesterol (LDL-C): LDL precipitation with dextran sulfate, CHOD-PAP and cholesterol determination (ImmunoGmbH/Boehringer Mannheim); uric acid: uricase-PAP (Boehringer Mannheim); creatinine: Jaffé (Boehringer Mannheim); serum glucose: GOD-PAP; insulin after an overnight fast: insulin RIA Coat-A-Count (DPC). A strict quality control was obligatory for all laboratory tests. To improve the normal distribution, the natural logarithms were used for triclyceride, insulin and glucose serum concentrations.

Structural Modeling. The aim of the structural model is to explain the observed correlation structure between the diagnostic variables adjusted for age, gender and body mass index by superposing partial correlations of the variables with common correlations and correlations with two hypothetical latent factors. All manifest variables were centered by the mean of the sample.

With the endogenous manifest variables

$$
\eta=\left(\mathrm{Y}_{1}, \ldots, \mathrm{Y}_{10}\right)^{\prime}
$$

(table 1) and the exogenous variables

$$
\xi=\left(\text { Age }, \text { Sex }, F^{(1)}, F^{(2)}, E_{1}, \ldots, E_{10}\right)^{\prime}
$$

the structural model results in the presentation of Bentler and Weeks [14]

$$
\eta=\beta \eta+\gamma \xi \text {. }
$$

The variables age and sex serve as adjustment of the endogenous variables. $\mathrm{F}^{(1)}$ and $\mathrm{F}^{(2)}$ are two latent variables, and $\mathrm{E}_{1}, \ldots, \mathrm{E}_{10}$ are the residual errors for the variables $Y_{1}, \ldots, Y_{10}$. The parameters $\beta$ and $\gamma$ of the model are estimated by the maximum likelihood method, and the final model was found by several steps of model selection with the aim of optimal model fit.

All laboratory parameters of our structural model and the two variables systolic blood pressure and waist-to-hip ratio were adjusted for age, sex and body mass index. Furthermore, the body mass index itself was adjusted for age and sex. In this way, age, sex and the hypothetical factors $\mathrm{F}^{(1)}$ and $\mathrm{F}^{(2)}$ are the only exogenous variables of the model, i.e. only these variables vary completely independently. A certain part $\mathrm{R}^{2}$ of the variance of all other variables results from the associations which are described in the model.

The greater $\mathrm{R}^{2}$ the stronger the observed variability of the parameter is predetermined by the remaining variables of the model. The remaining part $100 \%-\mathrm{R}^{2}$ expresses the part of the independent variability of a variable and is a measure of the information it contributes for the further evaluation of a patient. The observed correlations between the variables consist of associations which can be explained biochemically or physiologically (shown in the model by duplicate arrows) and of common correlations which arise by the latent factors. The development of our structural model is based on the results of an explorative maximum likelihood factor analysis for the variables used. Computations are carried out by the SAS CALIS procedure [15] using maximum likelihood methods.

In our model, we interpret the two postulated latent variables as unobservable factors which reflect initially unknown causes. In the second step, we try to identify these causes as genetic causes and/or environmental causes. These latent factors contribute to the observed variances and covariances of the variables. They have a bivariate standard normal distribution in the population. However, their least square estimations in the sample have a slightly smaller standard deviation and are not unbiased [16]. Of course, this model is based on theoretically justified associations between the variables and is only valid under the conditions of both statistical and theoretical evidence. Since different models of this kind can be set up with the same data, the inference has an exploratory character only. The statistical evidence of our model and of the estimated parameters results from several characteristics ( $p$ value for the whole model, $p$ values for single coefficients and covariances, sufficiently small and normally distributed residuals, rates of explained variances of the variables, goodness of fit indices).

\section{Step two: Estimations of Variance Components - Research} Design and Methods

The same sample as in step one was used. The sample is divided into pedigrees of 42 families with 308 members and 841 single persons. With segregation analyses, without using information about genotypes, the covariance matrices of the estimated scores from step one were separated for all members of each family into the genetic, environment and residual variance and covariance components. Here, we assume that variability in the two scores $\mathrm{F}^{(1)}$ and $\mathrm{F}^{(2)}$ is due to the summed effects of additive polygenes, environment factors shared by members of the same household, or of the same sibship, or of the same couple, and a residual effect specific to each individual which may reflect measurement errors in the original variables and errors in the structural model. Under these assumptions, the $2 n \times 2 n$ covariance matrix for a family with $\mathrm{n}$ members is

$$
\begin{aligned}
& \Omega=\left(\begin{array}{ll}
\sigma_{\mathrm{a} \mathrm{F}^{(1)}}^{2} & \sigma_{\mathrm{aF}} \mathrm{F}^{(1) \mathrm{F}^{(2)}} \\
\sigma_{\mathrm{aF}} \mathrm{F}^{(1)} \mathrm{F}^{(2)} & \sigma_{\mathrm{a} \mathrm{F}^{(2)}}^{2}
\end{array}\right) \otimes 2 \Phi_{(\mathrm{n} \times \mathrm{n})}+\left(\begin{array}{cc}
\sigma_{\mathrm{uF}^{(1)}}^{2} & \sigma_{\mathrm{uF}^{(1)} \mathrm{F}^{(2)}} \\
\sigma_{\mathrm{uF}^{(1)} \mathrm{F}^{(2)}} & \sigma_{\mathrm{uF}}^{2}
\end{array}\right) \otimes \mathrm{U}_{(\mathrm{n} \times \mathrm{n})} \\
& +\left(\begin{array}{c}
\sigma_{\mathrm{eF}^{(1)}}^{2} \sigma_{\mathrm{eF}^{(1)} \mathrm{F}^{(2)}} \\
\sigma_{\mathrm{eF}^{(1)} \mathrm{F}^{(2)}} \sigma_{\mathrm{eF}^{(2)}}^{2}
\end{array}\right) \otimes \mathrm{I}_{(\mathrm{n} \times \mathrm{n})},
\end{aligned}
$$

where $\sigma_{\mathrm{a}}^{2}(1)$ is the additive genetic variance of the score $\mathrm{F}^{(1)}, \sigma_{\mathrm{a}}^{2} \mathrm{~F}^{(2)}$ is the additive genetic variance of the score $\mathrm{F}^{(2)}, \sigma_{\mathrm{a}^{(1)} \mathrm{F}^{(2)}}$ is the additive genetic covariance between $\mathrm{F}^{(1)}$ and $\mathrm{F}^{(2)}, \sigma_{\mathrm{uF}}^{2}(1), \sigma_{\mathrm{uF}^{(2)}}^{2}, \sigma_{\mathrm{uF}^{(1)} \mathrm{F}^{(2)}}$ are the corresponding variance and covariance components for the environmental factor, and $\sigma_{\mathrm{eF}^{(1)}}^{2}, \sigma_{\mathrm{eF}^{(2)}}^{2}, \sigma_{\mathrm{eF}^{(1)} \mathrm{F}^{(2)}}$ are the corresponding residual variance and covariance components. $\otimes$ is the Kronecker product of matrices, $\Phi$ is the known $\mathrm{n} \times \mathrm{n}$ matrix of the kinship coefficients representing half the theoretical correlations between pedigree members, and $\mathrm{U}$ is the $\mathrm{n} \times \mathrm{n}$ matrix of indicators for a common household with $0=$ none and $1=$ yes.

Four further parameters were included in the model: the expected values of the variables $\mathrm{F}^{(1)}$ and $\mathrm{F}^{(2)}$ of all members of the 42 families and the corresponding expected values of all single individuals without family members in the sample. Thus, a possible bias from the family part of the sample compared with the part of single individuals is taken into consideration. Alternatively, an ascertainment correction for all index patients was used basing on conditional likelihood. This variant was dropped because the results did not prove different. The log likelihood for a family based on multivariate normal distribution of the outcome variable $y=\left(F_{1}^{(1)}, \ldots, F_{n}^{(1)}, F_{1}^{(2)}, \ldots\right.$, $\left.\mathrm{F}_{\mathrm{n}}^{(2)}\right)^{\prime}$ in all $\mathrm{n}$ members of a family is with the exception of noninformative terms

$$
\ln L \propto-\frac{1}{2} \ln \operatorname{det} \Omega-\frac{1}{2}(\mathrm{y}-\mathrm{A} \mu)^{\prime} \Omega^{-1}(\mathrm{y}-\mathrm{A} \mu) .
$$

The $2 \mathrm{n} \times 4$ matrix A denotes the indicators for the components of the mean $\mu=\left(\mu_{\mathrm{F}^{(1)} \text {,family }}, \mu_{\mathrm{F}^{(1)} \text {,single }} \mu_{\mathrm{F}^{(2)} \text {,family }}, \mu_{\mathrm{F}^{(2)} \text {,single }}\right)^{\prime}$ in the two parts of the sample, the part with all members of families and the part of single persons. Of course, the covariance matrix $\Omega$ collapses to a $2 \times 2$ matrix for a single person without data from his/her family, 


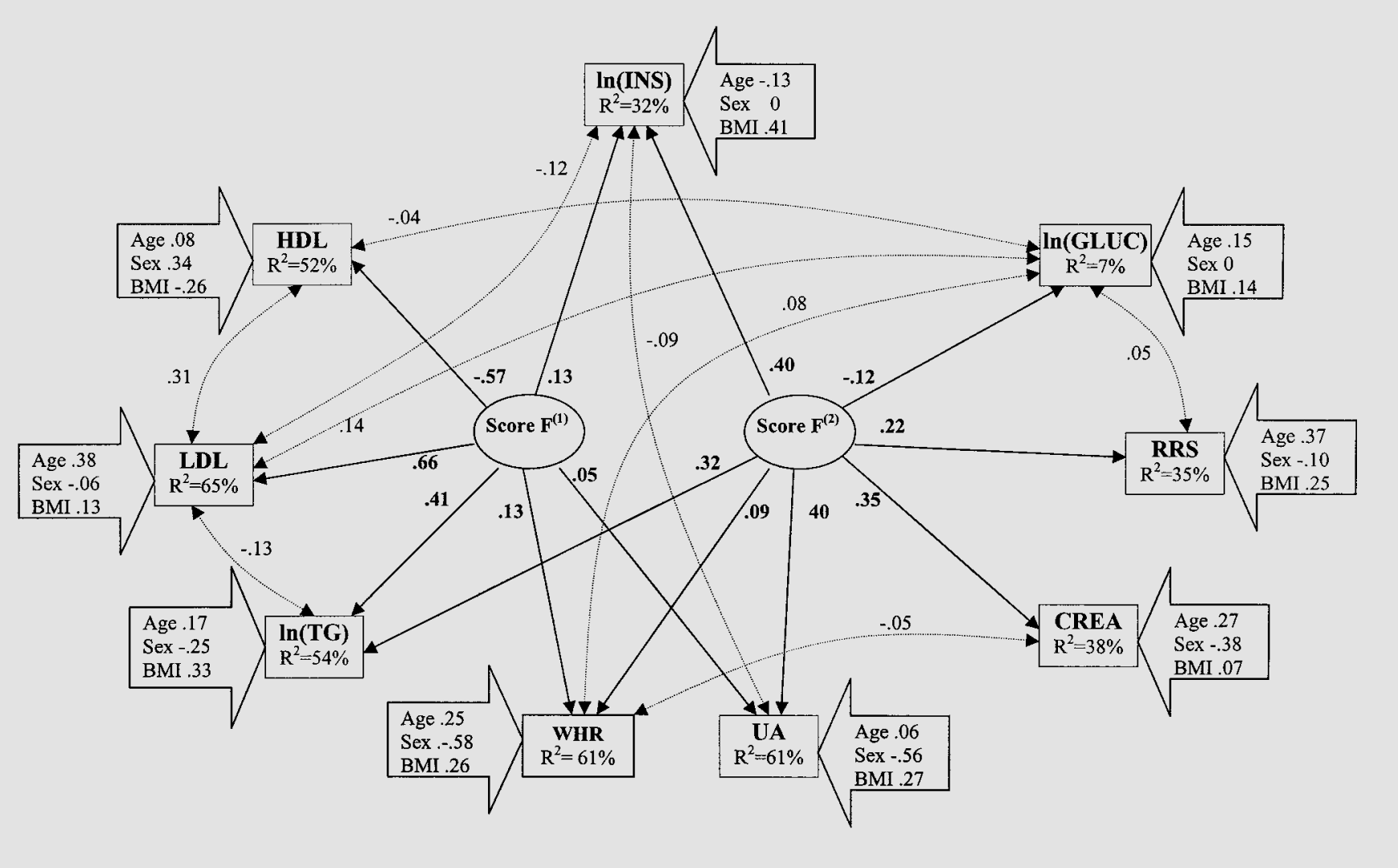

Fig. 1. Structural model with path coefficients for the variables log insulin [ln(INS)], log glucose [ln(GLUC)], creatinine (CREA), uric acid (UA), body mass index (BMI), log triglycerides [ $\ln (\mathrm{TG})]$, low-density cholesterol (LDL), high-density cholesterol (HDL), systolic blood pressure (RRS), waist-hip ratio (WHR), age and sex.

and such a person does not contribute information for the variance and covariance components but for the means only.

The maximum likelihood estimations of the model parameters are based on scoring iteration [9] and were carried out with our own SAS-IML [17] programs.

The standard errors of the estimated parameters result from asymptotic estimations from the inverse of the information matrix in the whole model.

\section{Results}

Figure 1 shows the structural model. The $\mathrm{p}$ value of the $\chi^{2}$ statistic for the model is 0.68 and, together with other characteristics, shows a sufficient fit of the model to the data (goodness of fit index GFI = 0.998; maximum absolute deviation of the correlation predicted by the model from the observed correlations 0.037 ; good correspondence of the residuals to normal distribution). Only few two-sided and no one-sided associations were taken into the model. Nevertheless, these few associations together with both the latent factors $\mathrm{F}^{(1)}$ and $\mathrm{F}^{(2)}$ are adequate to explain the remarkably high parts of variance and covariance of the variables. In this model, the variable LDL is especially strongly predetermined $\left(\mathrm{R}^{2}=65 \%\right)$. HDL $\left(\mathrm{R}^{2}=\right.$ $52 \%)$, triglycerides $\left(\mathrm{R}^{2}=54 \%\right)$, waist-to-hip ratio $\left(\mathrm{R}^{2}=\right.$ $61 \%)$ and uric acid $\left(\mathrm{R}^{2}=61 \%\right)$ show an explained high variance as well. The path coefficients of the latent factors to the original variables allow an interpretation of the factors. Thus, high factor values of $\mathrm{F}^{(1)}$ show pathologically increased LDL (path coefficient 0.66), triglycerides $(0.41)$, insulin $(0.13)$, waist-to-hip ratio $(0.13)$ and decreased HDL cholesterol $(-0.57)$. The known negative correlation between LDL and HDL is reproduced by the latent variable $\mathrm{F}^{(1)}$ but not by the bivariate residual correlation (path coefficient 0.31 ). Factor $\mathrm{F}^{(2)}$ reflects mainly increased uric acid (path coefficient 0.40 ), insulin (0.40) and triglycerides $(0.32)$. The body mass index can be explained by a $22 \%$ share of the variance of age and sex. 
The remaining $78 \%$ of the variance result from exogenous nonobserved sources. An influence of the two latent factors on the body mass index is excluded in this model. With the present data, other potential associations could not be shown by the model.

On the basis of this structural model, both score values can be estimated for each person by a simple computa-

Table 2. Regression coefficients for the estimation of the scores basing on the standardized original variables, and means and standard deviations of the estimated scores $\mathrm{F}^{(1)}$ and $\mathrm{F}^{(2)}$

\begin{tabular}{lrc}
\hline Variables & $\begin{array}{l}\text { Score } \\
\text { coefficient } \\
\text { for } \mathrm{F}^{(1)}\end{array}$ & $\begin{array}{l}\text { Score } \\
\text { coefficient } \\
\text { for } \mathrm{F}^{(2)}\end{array}$ \\
\hline $\begin{array}{l}\text { Triglycerides, } \mathrm{mmol} / \mathrm{l} \\
\quad \text { log triglycerides }\end{array}$ & 0.22 & 0.23 \\
HDL cholesterol, $\mathrm{mmol} / 1$ & -0.58 & 0.12 \\
LDL cholesterol, mmol/1 & 0.78 & -0.04 \\
Uric acid, $\mu \mathrm{mol} / 1$ & -0.05 & 0.52 \\
Creatinine, $\mu \mathrm{mol} / 1$ & -0.04 & 0.27 \\
Glucose, mmol/1 & & \\
$\quad$ log glucose & -0.13 & -0.07 \\
Insulin, nmol/1 & & \\
$\quad$ log insulin & 0.09 & 0.31 \\
Systolic blood pressure & -0.02 & 0.16 \\
Waist-to-hip ratio & 0.02 & 0.13 \\
Age & -0.23 & -0.18 \\
Sex & 0.26 & 0.50 \\
Body mass index, $\mathrm{kg} / \mathrm{m}^{2}$ & -0.33 & -0.39 \\
\hline Mean & 0.00 & 0.00 \\
Standard deviation & 0.97 & 0.74 \\
\hline
\end{tabular}

tional instruction. As expected, the scores of all persons in our sample built a scatterplot in regular form around the origin corresponding to a bivariate normal distribution. A clustering of the scores into distinct types is not observed.

Table 2 shows the score regression coefficients and means and standard deviations of the estimated scores $\mathrm{F}^{(1)}$ and $\mathrm{F}^{(2)}$. Table 3 contains the results of the estimation of the variance and covariance components for some different models. The Akaike information criterion preferred the full model. The model without covariances does not show a significant deterioration compared to the full model (likelihood ratio test $p=0.060$ ) and should be preferred for statistical reasons because of the theoretical absence of correlation between the two latent variables. Merely the least square estimation procedure of $F^{(1)}$ and $\mathrm{F}^{(2)}$ generated a correlation between the scores. Both models show nearly the same estimations of the variance and covariance components. In the models which include parameters for the means, the difference of the estimated means is surprisingly clear between the two parts of the sample, the part of family members and the part of single individuals. This difference may reflect a bias in the sampling procedure in favor of family members [18]. As hoped, one of the latent variables $\left(\mathrm{F}^{(1)}\right)$ shows a clear genetic effect measured by the additive genetic variance component. This is right in all models. Especially in the full model, the variance component for additive genetic effects is estimated at 0.454 , corresponding to a heritability of $42 \%$, and a negligible environmental effect. The other latent variable $\left(\mathrm{F}^{(2)}\right)$ shows low effects in both variance components only $(16 \%$ heritability and $7 \%$ environment).

Table 3. Bivariate analysis of the scores $\mathrm{F}^{(1)}$ and $\mathrm{F}^{(2)}$

\begin{tabular}{|c|c|c|c|c|c|c|c|c|c|c|c|c|c|c|}
\hline \multirow[t]{2}{*}{ Model } & \multicolumn{13}{|c|}{ Model parameters ${ }^{\mathrm{a}}$} & \multirow[t]{2}{*}{ Akaike ${ }^{b}$} \\
\hline & $\sigma_{\mathrm{aF} 1}^{2}$ & $\sigma_{\mathrm{aF} 2}^{2}$ & $\sigma_{\mathrm{aF} 1 \mathrm{~F} 2}$ & $\sigma_{\mathrm{uF} 1}^{2}$ & $\sigma_{\mathrm{uF} 2}^{2}$ & $\sigma_{\mathrm{uF} 1 \mathrm{~F} 2}$ & $\sigma_{\mathrm{eF} 1}^{2}$ & $\sigma_{\mathrm{eF} 2}^{2}$ & $\sigma_{\mathrm{eF} 1 \mathrm{~F} 2}$ & $\mu_{\mathrm{F} 1, \text { family }}$ & $\mu_{\mathrm{F} 1, \text { single }}$ & $\mu_{\mathrm{F} 2, \text { family }}$ & $\mu_{\mathrm{F} 2, \text { single }}$ & \\
\hline Complete & $\begin{array}{c}0.454 \\
(0.028)\end{array}$ & $\begin{array}{c}0.122 \\
(0.008)\end{array}$ & $\begin{array}{c}-0.080 \\
(0.008)\end{array}$ & $\begin{array}{c}0.000 \\
(0.006)\end{array}$ & $\begin{array}{c}0.050 \\
(0.002)\end{array}$ & $\begin{array}{c}0.025 \\
(0.002)\end{array}$ & $\begin{array}{c}0.543 \\
(0.013)\end{array}$ & $\begin{array}{c}0.350 \\
(0.004)\end{array}$ & $\begin{array}{c}0.140 \\
(0.004)\end{array}$ & $\begin{array}{c}0.027 \\
(0.006)\end{array}$ & $\begin{array}{c}0.264 \\
(0.003)\end{array}$ & $\begin{array}{l}-0.006 \\
(0.001)\end{array}$ & $\begin{array}{l}-0.100 \\
(0.001)\end{array}$ & -717.27 \\
\hline Without covariances & $\begin{array}{c}0.474 \\
(0.029)\end{array}$ & $\begin{array}{c}0.133 \\
(0.009)\end{array}$ & - & $\begin{array}{c}0.000 \\
(0.006)\end{array}$ & $\begin{array}{c}0.046 \\
(0.002)\end{array}$ & - & $\begin{array}{c}0.532 \\
(0.013)\end{array}$ & $\begin{array}{c}0.344 \\
(0.004)\end{array}$ & - & $\begin{array}{c}0.025 \\
(0.006)\end{array}$ & $\begin{array}{c}0.263 \\
(0.003)\end{array}$ & $\begin{array}{l}-0.006 \\
(0.001)\end{array}$ & $\begin{array}{l}-0.100 \\
(0.001)\end{array}$ & -737.19 \\
\hline Without means & $\begin{array}{c}0.467 \\
(0.029)\end{array}$ & $\begin{array}{c}0.200 \\
(0.009)\end{array}$ & $\begin{array}{c}-0.048 \\
(0.008)\end{array}$ & $\begin{array}{c}0.000 \\
(0.006)\end{array}$ & $\begin{array}{c}0.046 \\
(0.002)\end{array}$ & $\begin{array}{c}0.018 \\
(0.002)\end{array}$ & $\begin{array}{c}0.534 \\
(0.013)\end{array}$ & $\begin{array}{c}0.299 \\
(0.004)\end{array}$ & $\begin{array}{c}0.117 \\
(0.004)\end{array}$ & - & - & - & - & -736.55 \\
\hline $\begin{array}{l}\text { Without covariances } \\
\text { and means }\end{array}$ & $\begin{array}{c}0.478 \\
(0.029)\end{array}$ & $\begin{array}{c}0.206 \\
(0.009)\end{array}$ & - & $\begin{array}{c}0.000 \\
(0.006)\end{array}$ & $\begin{array}{c}0.044 \\
(0.002)\end{array}$ & - & $\begin{array}{c}0.529 \\
(0.013)\end{array}$ & $\begin{array}{c}0.294 \\
(0.004)\end{array}$ & - & - & - & - & - & -746.96 \\
\hline
\end{tabular}

Maximum likelihood estimations. Figures in parentheses are standard errors.

Akaike criterion $=\log$ likelihood minus number of estimated parameters. 


\section{Discussion}

The well-known dependence of laboratory parameters on age, sex and body mass index is quantified in the structural model by the path coefficients and by and large confirms our expectations. Alternatively, the body mass index could be considered as an endogenous variable. In this case the variable would be the result and not the cause of the metabolic syndrome. However, a structural model which reflects this idea could not be derived from our data.

The score $\mathrm{F}^{(1)}$ seems to be quite suitable as a quantitative phenotypic multivariate trait to search for genetic linkage with candidate genes or genetic markers. It reflects mainly triglycerides and both fractions of cholesterol and involves their high heritability (heritability of log triglycerides $24-31 \%$ [8], 27\% estimated using our own data; heritability of HDL 35\% and of LDL 29\% both estimated using our own data). Surprisingly, the score $F^{(1)}$ does not point out any environment component. $\mathrm{F}^{(2)}$ seems rather to be determined by both, genetic and environmental influences.

In conclusion, two hypothetical latent factors prove to be effective in the modeling of associations between adjusted laboratory parameters and findings in a structural model. One of these factors, which combines information mainly about cholesterol fractions and triglycerides, shows a comparatively high estimation value of the heritability and a disappeared environment component. Thus, it appears suitable as a quantitative phenotypic trait in linkage analyses for the search of genetic causes of the metabolic syndrome. The second factor $\mathrm{F}^{(2)}$ shows relatively low estimation values for variance components which are caused both by genetic and environmental effects. In contrast to estimated principal components, the estimated scores of this latent variable can be interpreted in a context of associations, caused by physiological factors.

\section{Acknowledgments}

This work was supported by the Bundesministerium für Bildung und Forschung (BMBF) (grant No. 01 ZZ 9604) under the supervision of the Deutsche Forschungsanstalt für Luft- und Raumfahrt e.V. Bonn (DLR). We thank the associate editor and referees for constructive reviews on early versions of the manuscript.

\section{References}

1 Liese AD, Mayer-Davis EJ, Haffner SM: Development of the multiple metabolic syndrome: An epidemiologic perspective. Epidemiol Rev 1998;20:157-172.

2 Porkka KVK, Nuotio I, Pajukanta P, Ehnholm Ch, Suurinkeroinen L, Syvänne M, Lehtimäki T, Lahdenkari AT, Lahdenperä S, Ylitalo K, Antikainen M, Perola M, Raitakari OT, Kovanen P, Viikari JSA, Peltonen L, Taskinen MR: Phenotype expression in familial combined hyperlipidemia. Atherosclerosis 1997;133:245253.

3 Ciampi A, Schiffrin A, Thiffault J, Quintal H, Weitzner G, Poussier P, Lalla D: Cluster analysis of an insulin-dependent diabetic cohort toward definition of clinical subtypes. J Clin Epidemiol 1990;43:701-715.

4 Edwards KL, Austin MA, Newman B, Mayer E, Krauss RM, Selby JV: Multivariate analysis of the insulin resistance syndrome in women. Arterioscler Thromb 1994;14:1940-1945.

5 Donahue RP, Bean JA, Donhaue RDC, Goldberg RB, Prineas RJ: Does insulin resistance unite the separate components of the insulin resistance syndrome? Arteriosclerosis Thromb Vasc Biol 1997; 17:2413-2417.
6 Rice T, Vogler GP, Laskarewski PM, Perry TS, Rao DC: Familial aggregation of lipids and lipoproteins in families ascertained through random and nonrandom probands in the Stanford Lipid Research Clinics Family Study. Am J Med Genet 1991;39:270-277.

7 Chan J, Cheung J, Lau E, Wooà J: The metabolic syndrome in Hong Kong Chinese. Diabetes Care 1996;19:953-958.

8 Boehnke M, Moll PP, Lange K, Weidmann WH, Kottke BA: Univariate and bivariate analyses of cholesterol and triglyceride levels in pedigrees. Am J Med Genet 1986;23:775-792.

9 Lange K: Mathematical and Statistical Methods for Genetic Analysis. New York, Springer, 1997.

10 Amos CI: Robust variance-components approach for assessing genetic linkage in pedigrees. Am J Hum Genet 1994;54:535-543.

11 Abschlussbericht des Förderprojektes Differenzierung des Arterioskleroserisikos bei kombinierter Hyperlipoproteinämie und Hypertonie. Dresden, Medical Faculty, Technical University of Dresden, 1996
12 Study Protocol Differenzierung des Arterioskleroserisikos bei kombinierter Hyperlipoproteinämie und Hypertonie. Dresden, Medical Academy, 1992.

13 Jaross W, Assmann G, Bergmann S, Schulte H, DRECAN Team: Comaprison of risk factors for coronary heart disease in Dresden and Münster. Eur J Epidemiol 1994;10:307-315.

14 Bentler PM, Weeks DG: Multivariate analysis with latent variables; in Krishnaiah PR, Kanal LN (eds): Handbook of Statistics. Amsterdam, North Holland Publishing Company, 1982, vol 2.

15 SAS/STAT: User's Guide. Cary, SAS Institute, 1992.

16 Harman HH: Modern Factor Analysis. Chicago, University of Chicago Press, 1976.

17 SAS/IML: User's Guide. Cary, SAS Institute, 1992.

18 Khoury MJ, Beaty TH, Cohen BH: Fundamentals of Genetic Epidemiology. New York, Oxford University Press, 1993. 